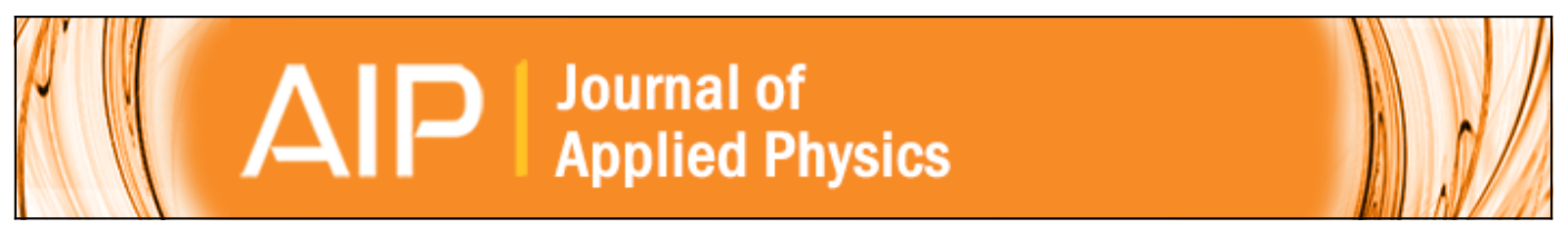

\title{
On the broadening of the magnetic entropy change due to Curie temperature distribution
}

Pablo Alvarez-Alonso, José L. Sánchez Llamazares, César F. Sánchez-Valdés, Gabriel J. Cuello, Victorino Franco, Pedro Gorria, and Jesús A. Blanco

Citation: Journal of Applied Physics 115, 17 A929 (2014); doi: 10.1063/1.4867346

View online: http://dx.doi.org/10.1063/1.4867346

View Table of Contents: http://scitation.aip.org/content/aip/journal/jap/115/17?ver=pdfcov

Published by the AIP Publishing

\section{Articles you may be interested in}

Textured Pr2Fe14B flakes with submicron or nanosize thickness prepared by surfactant-assisted ball milling J. Appl. Phys. 115, 17A734 (2014); 10.1063/1.4866879

Crystallographic alignment evolution and magnetic properties of $\mathrm{Nd}-\mathrm{Fe}-\mathrm{B}$ nanoflakes prepared by surfactantassisted ball milling

J. Appl. Phys. 111, 07A732 (2012); 10.1063/1.3679414

Optimization of melt spun RE-Fe-B powder composition for fully dense, high energy magnets

J. Appl. Phys. 109, 07 A742 (2011); 10.1063/1.3564969

Structure and magnetic properties of nanocrystalline PrCo 3

J. Appl. Phys. 107, 083916 (2010); 10.1063/1.3388364

Partitioning behavior and the effect of Co on the Curie temperature of nanocomposite PrFeCoB hard magnetic alloys

Appl. Phys. Lett. 79, 797 (2001); 10.1063/1.1388879

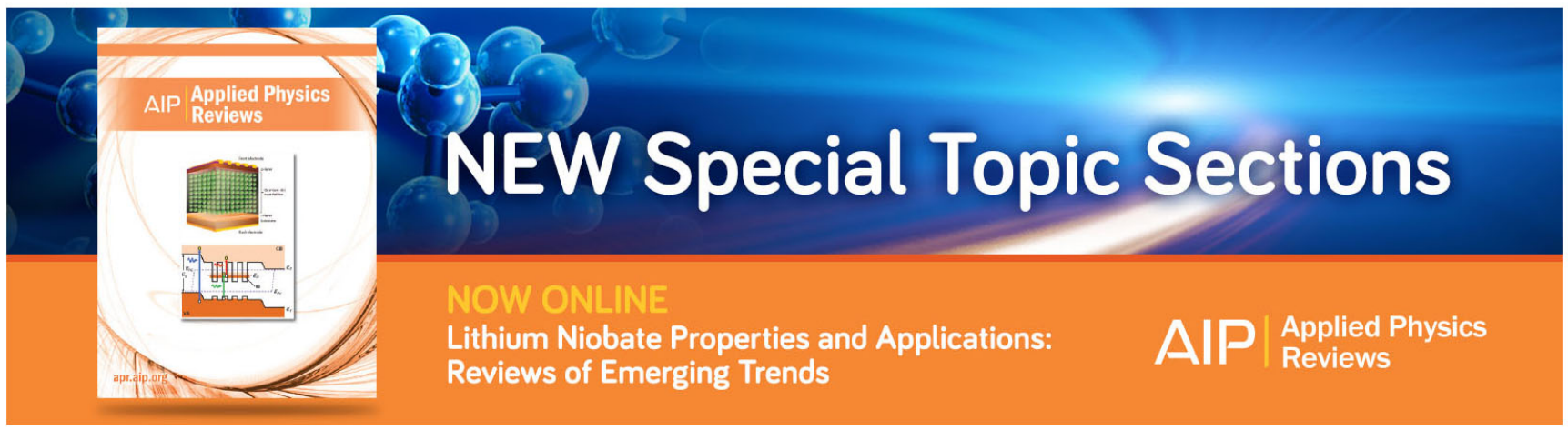




\title{
On the broadening of the magnetic entropy change due to Curie temperature distribution
}

\author{
Pablo Alvarez-Alonso, ${ }^{1}$ José L. Sánchez Llamazares, ${ }^{2}$ César F. Sánchez-Valdés, ${ }^{2}$ \\ Gabriel J. Cuello, ${ }^{3}$ Victorino Franco, ${ }^{4}$ Pedro Gorria, ${ }^{5, a)}$ and Jesús A. Blanco ${ }^{6}$ \\ ${ }^{1}$ Dept. Electricidad y Electrónica, Universidad del País Vasco, Barrio Sarriena s/n, 48940 Leioa, Spain \\ ${ }^{2}$ División de Materiales Avanzados, IPCyT, Camino a la presa San José 2055, 78216, San Luis Potosí, Mexico \\ ${ }^{3}$ Institute Laue Langevin, 6 rue Jules Horowitz, 38042 Grenoble, France \\ ${ }^{4}$ Dept. Física de la Materia Condensada, ICMSE-CSIC, Univ. Sevilla, P.O. Box 1065, 41080 Sevilla, Spain \\ ${ }^{5}$ Departamento de Física, EPI, Universidad de Oviedo, 33203 Gijón, Spain \\ ${ }^{6}$ Departamento de Física, Universidad de Oviedo, Calvo Sotelo s/n, 33007 Oviedo, Spain
}

(Presented 7 November 2013; received 23 September 2013; accepted 29 November 2013; published online 3 March 2014)

\begin{abstract}
We have studied the correlation between the broadening of the isothermal magnetic entropy change and the Curie temperature $\left(T_{\mathrm{C}}\right)$ distribution in nanostructured $\operatorname{Pr}_{2} \mathrm{Fe}_{17}$ and $\mathrm{Nd}_{2} \mathrm{Fe}_{17}$ alloys produced by high-energy ball-milling after milling times of 10,20 , and $40 \mathrm{~h}$. The changes in the microstructure affect the $\mathrm{Fe}$ local environments and as a consequence the magnetic interactions, giving rise to $T_{\mathrm{C}}$ distributions centered around $285 \mathrm{~K}$ and $330 \mathrm{~K}$ for the $\mathrm{Pr}_{2} \mathrm{Fe}_{17}$ and $\mathrm{Nd}_{2} \mathrm{Fe}_{17}$ alloys, respectively. The width of the distributions enlarges (up to $60 \mathrm{~K}$ ) as the milling-time increases, and consequently, the isothermal magnetic entropy change curves show an extended full width at half maximum. @ 2014 AIP Publishing LLC. [http://dx.doi.org/10.1063/1.4867346]
\end{abstract}

Magnetic refrigeration, the main application of the magneto-caloric (MC) effect, is potentially called to replace conventional vapor-cycle technology due to its higher energy efficiency and because it avoids green-house effect and depletion of ozone. ${ }^{1-3}$ Therefore, it is necessary to develop materials with large MC effect in broad temperature intervals around room temperature.

The MC materials usually exhibit useful working temperature intervals of about $20 \mathrm{~K},{ }^{4}$ under magnetic field changes of $2 \mathrm{~T}$, but often a refrigeration system demands a working temperature range that could reach $50 \mathrm{~K}$ or more. Thus, the increase of the temperature range without reducing their refrigerant capacity is a crucial aspect that MC materials must fulfill if their utilization in magnetic refrigeration technology is pursued. One of the material processing methods already used to enlarge the temperature range in which the maximum isothermal magnetic entropy change takes place is the mechanical milling of the MC material. ${ }^{5-7}$ In fact, the nanocrystallization of ferromagnetic $\mathrm{R}_{2} \mathrm{Fe}_{17}$ compounds $(\mathrm{R}=\mathrm{Pr}$ and $\mathrm{Nd})$ by means of ball milling has been proved as an illustrative example of this method, which leads to the enhancement of the refrigerant temperature range and the refrigerant capacity. ${ }^{8,9}$

Moreover, the Curie temperature $\left(T_{\mathrm{C}}\right)$ is strongly affected by the modification of the $\mathrm{Fe}-\mathrm{Fe}$ interatomic distances in a number of Fe-based alloys exhibiting magneto-volume anomalies. ${ }^{10}$ Changes in the material microstructure can produce local inhomogeneities around the $\mathrm{Fe}$ atoms, and a distribution of $\mathrm{Fe}-\mathrm{Fe}$ interatomic distances affecting the exchange interactions. The latter would affect the value of the $T_{\mathrm{C}}$ in the sense that instead of a unique value for the $T_{\mathrm{C}}$ a more reliable picture is to consider also a distribution function for this magnitude in

\footnotetext{
a) Author to whom correspondence should be addressed. Electronic mail: pgorria@uniovi.es.
}

the mechanically treated material. This distribution of $T_{\mathrm{C}}$ values can induce a broadening of the magnetic entropy change curves. In this paper, we report on the broadening of the isothermal magnetic entropy change and the Curie temperature distribution in ball-milled $\mathrm{Pr}_{2} \mathrm{Fe}_{17}$ and $\mathrm{Nd}_{2} \mathrm{Fe}_{17}$ compounds.

As-cast pellets and 10, 20, and $40 \mathrm{~h}$ ball-milled samples were fabricated following the procedure described elsewhere. ${ }^{11}$ Scanning (SEM) and transmission (TEM) electron microscopy were used to study the microstructure and morphology of the milled powders. Room temperature x-ray powder diffraction patterns were collected in a highresolution x-ray powder diffractometer (Seifert model $\mathrm{XRD} 3000)$ using graphite-monochromated $\mathrm{Cu} \mathrm{K} \alpha$ radiation $(\lambda=1.5418 \AA)$. Room temperature neutron powder diffraction patterns were collected on the D1B two-axis neutron diffractometer (ILL, Grenoble, France) using a neutron wavelength of $2.52 \AA$. Le Bail analyses were performed using the FullProf suite package. ${ }^{12}$

Magnetization vs. temperature curves under a constant applied magnetic field of $\mu_{\mathrm{o}} H=20 \mathrm{mT}$ in the temperature range $250-400 \mathrm{~K}$ were obtained in a Faraday balance. Isothermal $M\left(\mu_{0} H\right)$ curves were measured in two magnetometers, a Lakeshore VSM 7407 (85-420 K) and in a Quantum Design PPMS (200-400 K). The isothermal magnetic entropy change, $\Delta S_{\mathrm{M}}$, was determined through the appropriate Maxwell relation. ${ }^{13}$

SEM images reveal that the $\mathrm{Pr}_{2} \mathrm{Fe}_{17}$ and $\mathrm{Nd}_{2} \mathrm{Fe}_{17}$ milled powders are agglomerations of micronic grains $(0.5-10 \mu \mathrm{m})$ with rounded borders and narrower size distribution as the milling-time is increased. ${ }^{8,9}$ Higher magnification images obtained by TEM show grains formed by crystallites of nanometric size; the histograms describing the crystallite size distribution for the BM-10h and BM-20h compounds (powders ball-milled for 10 and $20 \mathrm{~h}$, respectively) follow log-normal functions with average size of the crystallites below $25 \mathrm{~nm}$ (see Table I). For the BM-40h samples, not enough crystallites 
TABLE I. Structural and magnetic data (the crystallographic parameters were obtained from the x-ray and neutron powder diffraction patterns).

\begin{tabular}{|c|c|c|c|c|c|c|c|c|}
\hline & \multicolumn{4}{|c|}{$\operatorname{Pr}_{2} \mathrm{Fe}_{17}$} & \multicolumn{4}{|c|}{$\mathrm{Nd}_{2} \mathrm{Fe}_{17}$} \\
\hline & Bulk & BM-10h & BM-20h & BM-40h & Bulk & BM-10h & BM-20h & BM-40h \\
\hline$a(\AA)$ & $8.584(1)$ & $8.580(1)$ & 8. 582(1) & $8.583(1)$ & $8.582(1)$ & $8.580(1)$ & $8.578(1)$ & $8.578(1)$ \\
\hline$b(\AA)$ & $12.460(1)$ & $12.455(1)$ & 12. $458(1)$ & $12.453(1)$ & $12.459(1)$ & $12.455(1)$ & $12.453(1)$ & $12.454(1)$ \\
\hline$\tau_{\text {TEM }}(\mathrm{nm})$ & $\ldots$ & $23(1)$ & $18(1)$ & * & $\ldots$ & $17(3)$ & $10(2)$ & $\ldots$ \\
\hline$\tau_{\text {Diff }}(\mathrm{nm})$ & $\ldots$ & $20(1)$ & $17(1)$ & 11(1) & $\ldots$ & $18(1)$ & $15(1)$ & 11(1) \\
\hline $\bar{T}_{C}(\mathrm{~K})$ & $286(2)$ & $290(5)$ & $290(10)$ & $290(10)$ & $339(2)$ & $340(10)$ & $340(10)$ & $340(10)$ \\
\hline
\end{tabular}

appeared in the TEM images to obtain reliable statistical information. The upper right inset in Fig. 1 shows a representative TEM image of $\mathrm{Nd}_{2} \mathrm{Fe}_{17}$ BM-40h powders.

In Fig. 1, the room temperature neutron powder diffraction patterns corresponding to the $\mathrm{Nd}_{2} \mathrm{Fe}_{17}$ samples are depicted. All the intensity peaks observed in the pattern of the starting bulk alloy correspond to Bragg reflections belonging to a $\mathrm{Nd}_{2} \mathrm{Fe}_{17}$ phase with the rhombohedral $\mathrm{Th}_{2} \mathrm{Zn}_{17}$-type crystal structure; the estimated lattice parameters (see Table I) agree with those previously reported. ${ }^{14}$ The ball-milling process maintains the $\mathrm{Th}_{2} \mathrm{Zn}_{17}$-type crystal structure with almost unchanged values of the cell parameters, but alters the microstructure (see Table I). Details of the followed procedure are described elsewhere. ${ }^{15}$

Figure 2 shows the magnetization as a function of the temperature, $M(T)$ curves. Those curves were composed with the points corresponding to $\mu_{\mathrm{o}} H=1 \mathrm{~T}$ of the isothermal $M\left(\mu_{\mathrm{o}} H\right)$ measurements. Notice how the shape of the $M(T)$ curves changes as the milling-time increases: the ferro-to-paramagnetic phase transition becomes poorly defined. However, the position of the $\mathrm{d} M / \mathrm{d} T$ vs. $T$ curve minima (see insets in Figure 2) remains almost unaltered (see Table I). The latter can be attributed to the effect of a change in the local environment of $\mathrm{Fe}$ atoms at the grain boundaries, which

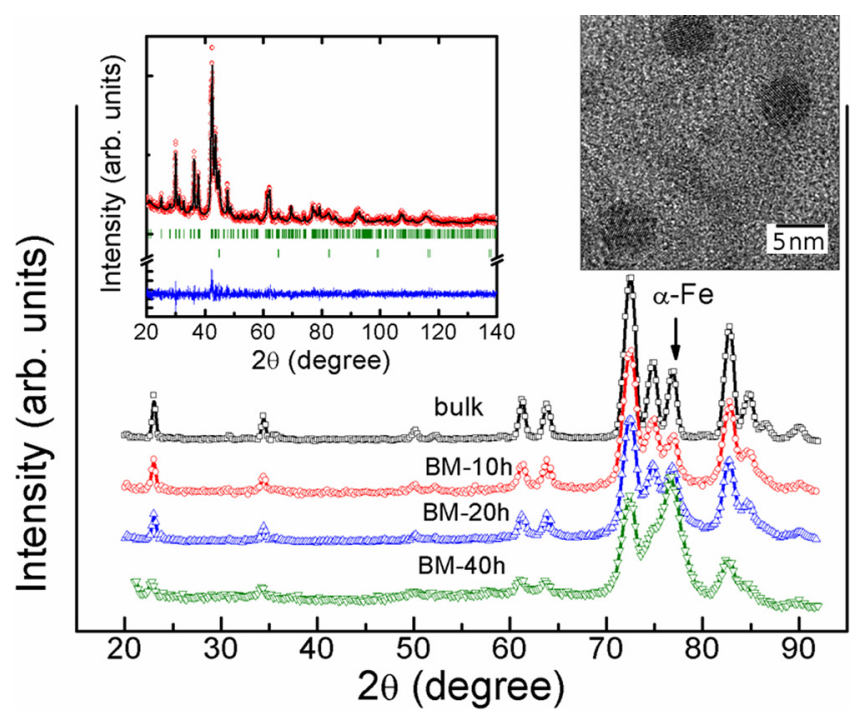

FIG. 1. Room temperature neutron powder diffraction patterns corresponding to bulk, BM-10h, BM-20h, and BM-40h $\mathrm{Nd}_{2} \mathrm{Fe}_{17}$ alloys. Upper left inset: Le Bail fit of the $\mathrm{x}$-ray powder diffraction pattern collected for $\mathrm{BM}-20 \mathrm{~h} \mathrm{Pr}_{2} \mathrm{Fe}_{17}$ sample. Upper right inset: TEM image of the BM-40h $\mathrm{Nd}_{2} \mathrm{Fe}_{17}$ sample. modifies the magnetic interactions ${ }^{16}$ and, thus, the Curie temperature of certain regions. Hence, we could picture the nanostructured powders having a distribution of $\mathrm{Fe}-\mathrm{Fe}$ interatomic distances around the value for the Bulk alloys giving rise to a distribution of Curie temperature values. ${ }^{8,9}$ Then, we describe the temperature dependence of the magnetization $M(T)$ by a superposition of individual power-law functions, according to ${ }^{17}$

$$
M(T)=m_{0} \int_{T_{C}}\left(\frac{T_{C}-T}{T_{C}}\right)^{\beta} \theta\left(T_{C}-T\right) \rho\left(T_{C}, \alpha\right) d T_{C} .
$$

Here, $m_{0}$ is a factor proportional to the saturation magnetization, $\beta$ is the temperature critical exponent of the magnetization, $\theta(x)$ is the Heavyside function (that ensures vanishing magnetization values at $\left.T=T_{\mathrm{C}}\right)$, and $\rho\left(T_{\mathrm{C}}, \alpha\right)$ a sample-specific $T_{\mathrm{C}}$ distribution function with parameters set $\alpha$. For the present case, we have assumed a Gaussian distribution

$$
\rho\left(T_{C}, \overline{T_{C}}, \Delta T_{C}\right)=\frac{1}{\sqrt{2 \pi} \Delta T_{C}} \exp \left(-\frac{1}{2} \frac{\left(T_{C}-\overline{T_{C}}\right)}{\Delta T_{C}^{2}}\right)
$$

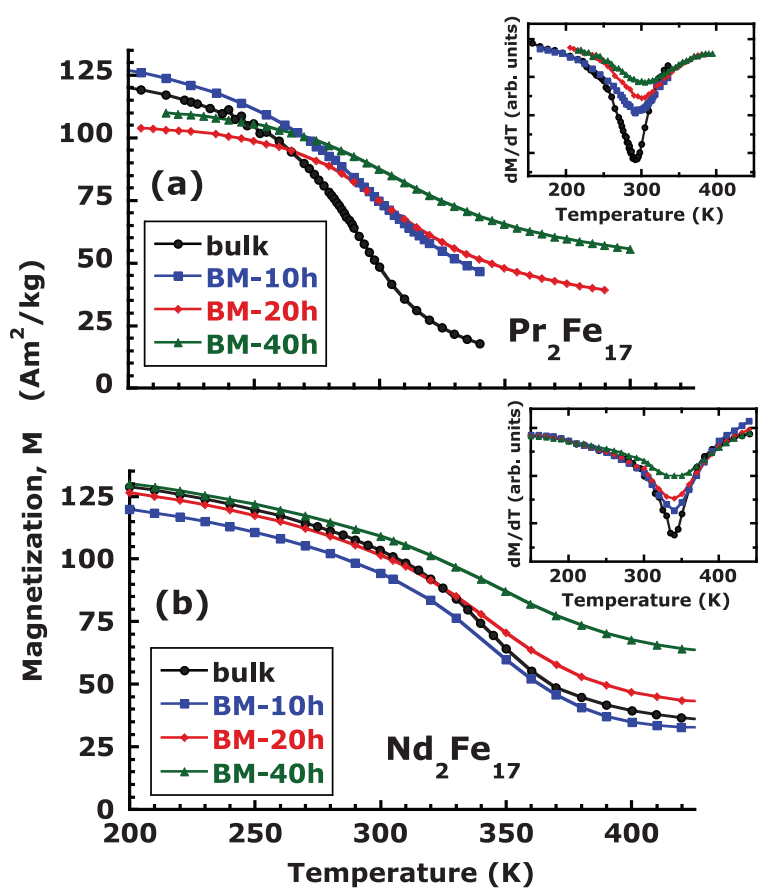

FIG. 2. Temperature dependence of the magnetization under an applied magnetic field of $1 \mathrm{~T}$ for the bulk and $\mathrm{BM}$ samples, (a) $\mathrm{Pr}_{2} \mathrm{Fe}_{17}$ and (b) $\mathrm{Nd}_{2} \mathrm{Fe}_{17}$. The two insets show the temperature derivatives of the magnetization. 


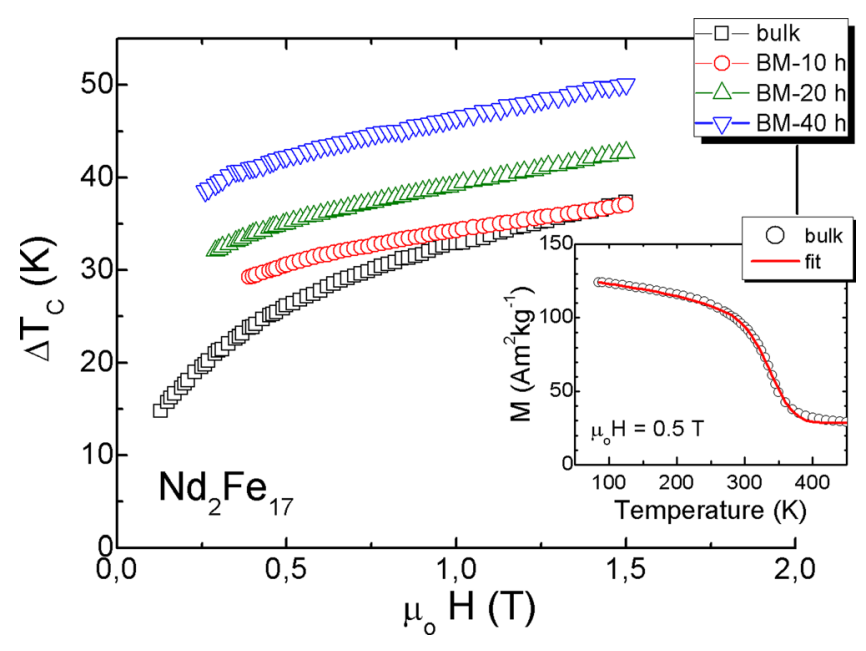

FIG. 3. Magnetic field dependence of the $\Delta T_{\mathrm{C}}$ for bulk and $\mathrm{BM} \mathrm{Nd_{2 }} \mathrm{Fe}_{17}$ samples. Inset: temperature dependence of the magnetization for the bulk $\mathrm{Nd}_{2} \mathrm{Fe}_{17}$ alloy (see text for details).

being $\bar{T}_{C}$ and $\Delta T_{\mathrm{C}}$ the average value and width of the $T_{\mathrm{C}}$ distribution.

Although the value of $T_{\mathrm{C}}$ is unique for any ferromagnetic single-phase alloy and this value must be determined under zero applied magnetic field, if the minimum of the $\mathrm{d} M / \mathrm{d} T$ vs. $T$ curves is chosen as the value for the $T_{\mathrm{C}}$ of the material, such minimum of the $\mathrm{d} M / \mathrm{d} T(T)$ curve exhibits a broadening as the applied magnetic field is increased. The inset in Fig. 3 shows the fit obtained for the temperature dependence of the magnetization in bulk $\mathrm{Nd}_{2} \mathrm{Fe}_{17}$ alloy for $\mu_{\mathrm{o}} H=0.5 \mathrm{~T}$ following this approach. The $T_{\mathrm{C}}$ distribution width (see Figure 3) augments with milling-time for each magnetic field. The magnetic field dependence of the width follows a power law, $\Delta T_{C}(H)=\Delta T_{C}^{0}+c H^{1 / \eta}$, with $\eta$ related with the critical exponents of the transition, as Berger et al. have shown. ${ }^{17}$ The fit to a power law gives for $\mathrm{Nd}_{2} \mathrm{Fe}_{17}$ : $\eta^{\text {Bulk }}=1.9 \pm 0.2, \quad \eta^{\text {BM-10h }}=3.0 \pm 0.3, \quad \eta^{\text {BM-20h }}=2.4 \pm 0.2$, and $\eta^{\mathrm{BM}-40 \mathrm{~h}}=2.8 \pm 0.3$. The differences in these values indicate variations in the magnetic interactions of the alloys and how they are affected by the applied magnetic field.

Fig. 4 plots the temperature dependence of the magnetic entropy change at $\mu_{\mathrm{o}} H=1.5 \mathrm{~T}$, for the studied samples. The temperatures at which the curves reach their maximum are similar for the four samples $\left(\sim T_{\mathrm{C}}\right)$. However, for the milled samples the maximum values of $\left|\Delta S_{\mathrm{M}}\right|$ diminish with the milling-time because the drop of the magnetization is less pronounced as milling-time increases.

In summary, ball-milled $\operatorname{Pr}_{2} \mathrm{Fe}_{17}$ and $\mathrm{Nd}_{2} \mathrm{Fe}_{17}$ alloys maintain the $\mathrm{Th}_{2} \mathrm{Zn}_{17}$-type crystal structure but their crystallite size is reduced below $25 \mathrm{~nm}$. The local environment of $\mathrm{Fe}$ atoms seems to be altered during the nanocrystallization

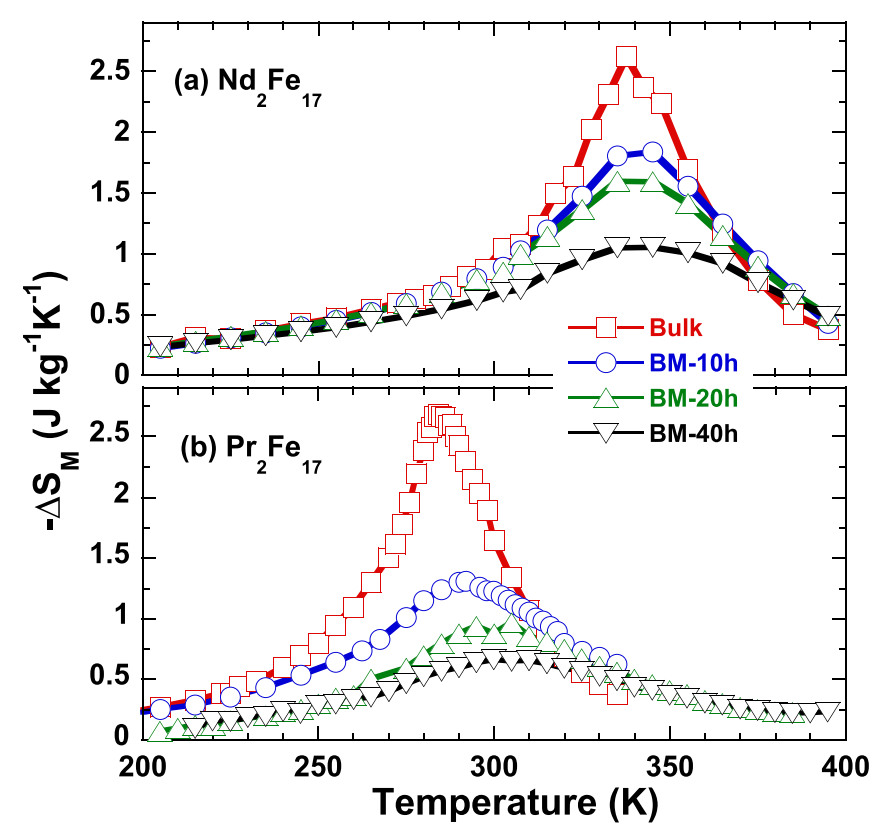

FIG. 4. Temperature dependence of the magnetic entropy change under $\mu_{\mathrm{o}} \Delta H=1.5 \mathrm{~T}$ for the bulk and $\mathrm{BM} \mathrm{Pr}_{2} \mathrm{Fe}_{17}$ and $\mathrm{Nd}_{2} \mathrm{Fe}_{17}$ samples.

process, giving rise to a Curie temperature distribution around the bulk's value. Fitting the magnetization vs. temperature curves with a Gaussian-type Curie temperature distribution, we found that the longer milling, the wider the $T_{\mathrm{C}}$ distribution, which correlates to the enlargement of the magnetic entropy change full width at half maximum.

We thank the financial support from MICINN, Spain (project MAT2011-27573-C04) and from CONACYT, Mexico (CB-2010-01-156932). J.L.S.Ll. thanks the support received from LINAN, IPICyT. We thank ILL and CRGD1B, the SCTs at the University of Oviedo, and the electron microscopy service of the IPICYT.

\footnotetext{
${ }^{1}$ V. K. Pecharsky and J. Gschneidner, Int. J. Refrig. 29, 1239 (2006).

${ }^{2}$ A. Tishin, J. Magn. Magn. Mater. 316, 351 (2007).

${ }^{3}$ V. Franco et al., Annu. Rev. Mater. Res. 42, 305 (2012).

${ }^{4}$ K. Gschneidner et al., Scr. Mater. 67, 572 (2012).

${ }^{5}$ B. Chevalier et al., Appl. Phys. A 80, 601 (2005).

${ }^{6}$ P. Gorria et al., J. Phys. D: Appl. Phys. 41, 192003 (2008).

${ }^{7}$ J. Gass et al., J. Appl. Phys. 103, $07 \mathrm{~B} 309$ (2008).

${ }^{8}$ P. Gorria et al., Acta Mater. 57, 1724 (2009).

${ }^{9}$ P. Álvarez et al., J. Phys.: Condens. Matter 22, 216005 (2010).

${ }^{10}$ P. Álvarez-Alonso et al., Phys. Rev. B 86, 184411 (2012).

${ }^{11}$ P. Alvarez et al., J. Non-Cryst. Solids 354, 5172 (2008).

${ }^{12}$ J. Rodríguez-Carvajal, Physica B 192, 55 (1993).

${ }^{13}$ P. Álvarez et al., J. Alloys Compd. 504, S150 (2010).

${ }^{14}$ G. Long et al., J. Appl. Phys. 76, 5383 (1994).

${ }^{15}$ D. Martinez-Blanco et al., J. Phys.: Condens. Matter 20, 335213 (2008).

${ }^{16}$ G. Campillo et al., J. Magn. Magn. Mater. 237, 61 (2001).

${ }^{17}$ A. Berger et al., J. Appl. Phys. 91, 8393 (2002).
} 